\title{
ITERATIVE BOUNDARY ELEMENT METHOD FOR CRACKS IN A THERMAL MEDIUM WITH EXACT CRACK FACE BOUNDARY CONDITIONS
}

\author{
QIAOYUN ZHANG ${ }^{1,3}$, YAGUANG GUO ${ }^{1} \&$ MINGHAO ZHAO ${ }^{1,2,3}$ \\ ${ }^{1}$ School of Mechanics and Engineering Science, Zhengzhou University, China \\ ${ }^{2}$ School of Mechanical Engineering, Zhengzhou University, China \\ ${ }^{3}$ Henan Key Engineering Laboratory for Anti-Fatigue Manufacturing Technology, Zhengzhou University, China
}

\begin{abstract}
An iterative boundary element method is proposed to analyze cracks with exact crack face boundary conditions in thermoelastic solids. Herein, the crack was opened under external loading, whereupon the opened cavity was considered a domain. The boundary element method for the crack-cavity domain and the sub-region boundary element method for the outer thermoelastic solid were used iteratively to obtain the real deformed crack faces. In this approach, the exact face boundary conditions on crack faces were used and the stress intensity factor and thermal flux density intensity factor near the crack tip were calculated.
\end{abstract}

Keywords: thermoelastic solid, boundary element method, crack, exact boundary conditions, stress intensity factor, thermal flux density intensity.

\section{INTRODUCTION}

Thermoelastic solids are sensitive to the presence of cracks, which cause a thermal flux concentration and affect the reliability and working life of materials and structures. Therefore, fracture problems in thermoelastic solids have attracted much attention [1]-[8].

Owing to the thermo-mechanical coupling property and the complex crack face boundary conditions in thermoelastic solids, it is difficult to find analytical solutions for some fracture problems, and thus numerical methods are adopted to study these problems [9]-[12]. Among these numerical methods, the boundary element method (BEM) [13], [14] reduces the solving dimensions and exhibits high accuracy, and is therefore very efficient to solve fracture problems in thermoelastic solids. Prasad et al. have studied several thermoelastic crack problems using dual BEM, wherein the stress intensity factors were obtained according to the independent $J$-integral [15]. Ekhlakov et al. have analyzed thermoelastic cracks in functionally-graded materials by BEM and have studied the influence that the material gradation, thermo-mechanical coupling, crack orientation and thermal shock loading have on the fracture behavior [16]. Pasternak has studied the inclined-crack model, edge crack model, the two-parallel-crack model and the kinked-crack model in two-dimensional anisotropic thermoelastic solids using BEM [17]. Finally, Zhao et al. have studied the extended intensity factors of an elliptical crack in isotropic three-dimensional thermoelastic solids [18].

Up to now, studies of thermal crack models have almost entirely been based on thermallyimpermeable or -permeable crack face boundary conditions, but these two boundary conditions are too limiting for most cases. Zhong and Kang [19] and Zhong et al. [20] have therefore proposed a new partially-thermally-permeable crack model, which demonstrates that the crack face boundary conditions of the thermal crack model significantly affect the fracture behavior. In the present work, we consider the exact crack face boundary conditions for a thermal-medium crack model, and an iterative BEM to solve this nonlinear crack model is proposed. The paper is organized as follows: In Section 2, the basic equations for thermoelastic solids are given. In Section 3, the iterative BEM is proposed for a central crack 
model in thermoelastic solids. In Section 4, numerical results are presented to demonstrate the relationships between the stress and thermal flux density intensity factors and the external mechanical and thermal loadings. Finally, in Section 5 the main conclusions are drawn.

\section{BASIC EQUATIONS}

In the absence of a body force or internal heat source, the governing equations for isotropic thermoelastic solids in the $O x z$ cartesian coordinate system are given by

$$
\begin{aligned}
& \sigma_{i j, i}=0, \\
& h_{i, i}=0,
\end{aligned} \quad(i, j=1,2 \text { or } x, z)
$$

where $\sigma_{i j}$ is the stress component and $h_{i}$ is the thermal flux component. The constitutive equations are given by [1]

$$
\begin{aligned}
& \sigma_{x}=2 G \frac{\partial u}{\partial x}+\frac{E v}{(1+v)(1-2 v)}\left(\frac{\partial u}{\partial x}+\frac{\partial w}{\partial z}\right)-\frac{E}{1-2 v} \alpha \theta, \\
& \sigma_{z}=2 G \frac{\partial w}{\partial z}+\frac{E v}{(1+v)(1-2 v)}\left(\frac{\partial u}{\partial x}+\frac{\partial w}{\partial z}\right)-\frac{E}{1-2 v} \alpha \theta, \\
& \tau_{x y}=G\left(\frac{\partial u}{\partial z}+\frac{\partial w}{\partial x}\right), \\
& h_{x}=-\beta \frac{\partial \theta}{\partial x}, \quad h_{z}=-\beta \frac{\partial \theta}{\partial z},
\end{aligned}
$$

where $E$ is the elasticity modulus, $G$ is the shear modulus, $v$ is the Poisson ratio, $\theta$ is the temperature, $\alpha$ is the thermal expansion coefficient and $\beta$ is the heat conductivity. Substituting eqn (2) into eqn (1), the governing equations can be rewritten as

$$
\begin{aligned}
& 2 G \frac{\partial^{2} u}{\partial x^{2}}+\frac{E v}{(1+v)(1-2 v v)}\left(\frac{\partial^{2} u}{\partial x^{2}}+\frac{\partial^{2} w}{\partial x \partial z}\right)+\frac{E}{1-2 v} \alpha \frac{\partial \theta}{\partial x}+G\left(\frac{\partial^{2} u}{\partial z^{2}}+\frac{\partial^{2} w}{\partial x \partial z}\right)=0 \\
& 2 G \frac{\partial^{2} u}{\partial z^{2}}+\frac{E v}{(1+v)(1-2 v)}\left(\frac{\partial^{2} u}{\partial x \partial z}+\frac{\partial^{2} w}{\partial z^{2}}\right)+\frac{E}{1-2 v} \alpha \frac{\partial \theta}{\partial z}+G\left(\frac{\partial^{2} u}{\partial x \partial z}+\frac{\partial^{2} w}{\partial x^{2}}\right)=0 \\
& -\beta\left(\frac{\partial^{2} \theta}{\partial x^{2}}+\frac{\partial^{2} \theta}{\partial z^{2}}\right)=0 .
\end{aligned}
$$

On the outer boundary of the solid, the displacement and surface traction boundary conditions are given by

$$
\begin{aligned}
& u_{i}=\bar{u}_{i} \text { on } \Gamma_{u}, \\
& t_{i}=\sigma_{i j} n_{j}=\bar{t}_{i} \text { on } \Gamma_{t},
\end{aligned}
$$

where $\Gamma_{u}$ and $\Gamma_{t}$ represent the boundaries with prescribed displacements and prescribed tractions, respectively. For the temperature field, the boundary conditions are given by

$$
\begin{aligned}
& \theta=\bar{\theta} \text { on } \Gamma_{\theta}, \\
& q=h_{i} n_{i}=\bar{q} \text { on } \Gamma_{q},
\end{aligned}
$$

where $\Gamma \theta$ and $\Gamma$ q represent the boundary with prescribed temperature and prescribed thermal flux density, respectively; $\bar{u}_{i}, \overline{t_{i}}, \bar{\theta}$ and $\bar{q}$ are the prescribed displacement, traction, 
temperature and thermal flux density, respectively; and ni is the outward normal vector. Note that $\Gamma \mathrm{u}+\Gamma \mathrm{t}=\Gamma \theta+\Gamma \mathrm{q}=\Gamma$.

\section{BOUNDARY ELEMENT METHOD FOR THERMOELASTIC SOLIDS WITH A CENTRAL CRACK}

Consider a thermoelastic plate with a central crack, as shown in Fig. 1 . The plate length is $2 l$ and height is $2 h$, and the crack length is $2 c$. The symmetric mechanical loading $p_{0}$ and thermal loading $q_{0}$ are applied on the upper and lower surface of the plate, while the crack faces are load-free. Under the applied mechanical and thermal loadings, the crack opens and the crack cavity $\Omega_{c}$ is formed. The boundary of the crack cavity can be expressed as

$$
\Gamma_{c}=\Gamma_{c 1}+\Gamma_{c 2},
$$

where $\Gamma_{c 1}$ and $\Gamma_{c 2}$ are the upper and lower faces of the crack cavity, respectively. Therefore, we adopted the sub-region BEM to divide the entire plate into the three domains $\Omega_{1}, \Omega_{2}$ and $\Omega_{c}$, as shown in Fig. 2. The boundaries of $\Omega_{1}$ and $\Omega_{2}$ can be expressed as:

$$
\begin{aligned}
& \Gamma_{1}=\Gamma_{12}+\Gamma_{1 c}+B_{1}+B_{2}+B_{3}, \\
& \Gamma_{2}=\Gamma_{21}+\Gamma_{2 c}+S_{1}+S_{2}+S_{3},
\end{aligned}
$$

where the divided regions are shown in Fig. 2. In the cavity of the opening crack, there exists only the temperature field, whose governing equation is yet the heat conduction equation, given as

$$
-\beta\left(\frac{\partial^{2} \theta^{c}}{\partial x^{2}}+\frac{\partial^{2} \theta^{c}}{\partial z^{2}}\right)=0
$$

where $\theta^{c}$ is the temperature field in the crack cavity.

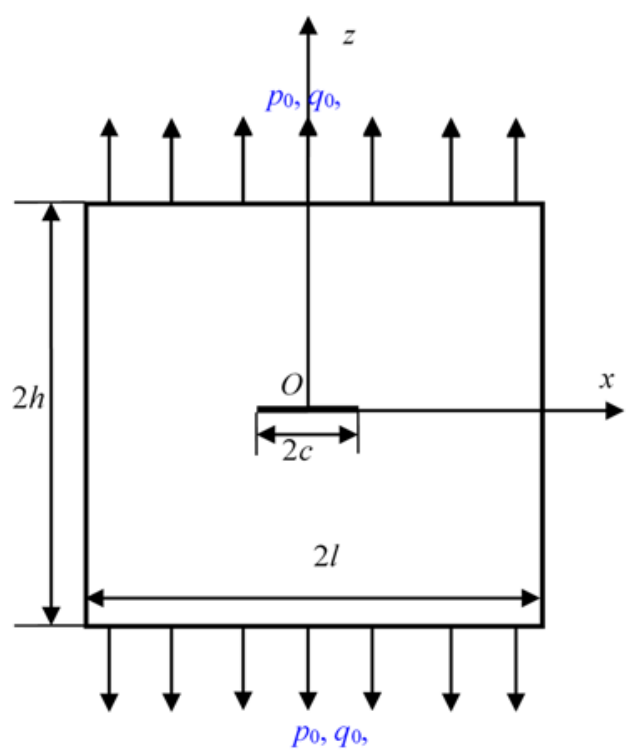

Figure 1: Schematic of a central crack in a thermoelastic rectangular plate under mechanical loading $p_{0}$ and thermal loading $q_{0}$. 


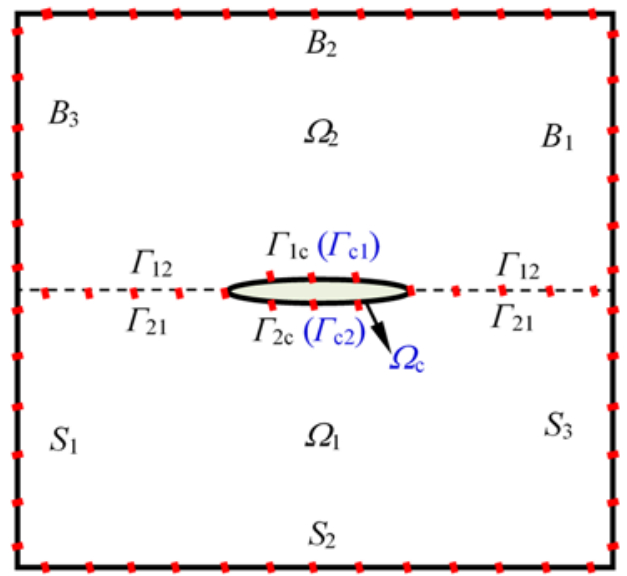

Figure 2: Schematic of the sub-regions of the central crack model and discretization of the involved boundaries.

The boundary conditions are given as follows:

(1) On the outer boundaries $B_{1}, B_{2}, B_{3}, S_{1}, S_{2}$ and $S_{3}$, the boundary conditions are

$$
\begin{array}{ll}
t_{1}=0, & t_{2}=p_{0}, \quad q=q_{0}, \quad(|x| \leq l, z=h), \\
t_{1}=0, & t_{2}=-p_{0}, \quad q=-q_{0}, \quad(|x| \leq l, z=-h), \\
t_{1}=0, & t_{2}=0, \quad q=0, \quad(x= \pm l,|z| \leq h),
\end{array}
$$

where $t_{i}(i=x, z)$ denotes the mechanical traction, $p_{0}$ is the external mechanical loading along the $z$-direction, and $q_{0}$ is the external thermal loading.

(2) On the common boundaries $\Gamma_{12}$ and $\Gamma_{21}$ between sub-regions $\Omega_{1}$ and $\Omega_{2}$, along the $x$-axis, the boundary conditions are

$$
\begin{aligned}
& \left.u\right|_{\Gamma_{12}}=\left.u\right|_{\Gamma_{21}},\left.\quad w\right|_{\Gamma_{12}}=\left.w\right|_{\Gamma_{21}}, \\
& \left.t_{1}\right|_{\Gamma_{12}}=-\left.t_{1}\right|_{\Gamma_{21}},\left.\quad t_{2}\right|_{\Gamma_{12}}=-\left.t_{2}\right|_{\Gamma_{21}}, \quad(c \leq|x| \leq l, z=0) . \\
& \left.\theta\right|_{\Gamma_{12}}=\left.\theta\right|_{\Gamma_{21}},\left.\quad q\right|_{\Gamma_{12}}=-\left.q\right|_{\Gamma_{21}},
\end{aligned}
$$

(3) On the common boundaries $\Gamma_{1 \mathrm{c}}$ and $\Gamma_{\mathrm{c} 1}\left(\Gamma_{2 \mathrm{c}}\right.$ and $\left.\Gamma_{\mathrm{c} 2}\right)$ between sub-regions $\Omega_{1}$ and $\Omega_{\mathrm{c}}\left(\Omega_{2}\right.$ and $\left.\Omega_{\mathrm{c}}\right)$, the boundary conditions are

$$
\begin{aligned}
& t_{1}=0, \quad t_{2}=0, \\
& \left.\theta\right|_{\Gamma_{\alpha c}}=\left.\theta\right|_{\Gamma_{c \alpha}},\left.\quad q\right|_{\Gamma_{\alpha c}}=-\left.q\right|_{\Gamma_{c \alpha}}, \quad(x, z) \in \Gamma_{c}, \quad \alpha=1,2 .
\end{aligned}
$$

We note that the thermal boundary conditions on the crack faces described in eqn (11) are the exact boundary conditions depending on the deformed crack faces.

\subsection{Iterative approach}

Owing to the existence of a temperature field in the crack cavity, the thermal flux density boundary condition on the upper and lower crack surfaces is affected by the deformed crack 
faces. This problem is a typical nonlinear problem that we propose to solve using an iterative BEM, whose iterative approach is described in detail in the following.

(1) The crack is initially treated as a slit that is considered to be thermally impermeable. Thus, the crack face boundary conditions are given by

$$
t_{1}=0, \quad t_{2}=0, \quad q=0 .
$$

In this case, only the two regions $\Omega_{1}$ and $\Omega_{2}$ remain in the thermoelastic plate. Thus, using the sub-region BEM and the boundary conditions in eqns (9), (10), and (12), the extended displacement fields $\left(u_{i}^{(1)}, \theta^{(1)}\right)$ and a new crack cavity region $\Omega_{\mathrm{c}}{ }^{(1)}$ are obtained.

(2) Next, the crack cavity region $\Omega_{\mathrm{c}}{ }^{(1)}$ is treated as a single domain and the temperatures $\theta^{1)}$ on the crack faces obtained in Step (1) are taken as the boundary conditions. Using the single-domain BEM, new values of the thermal flux density $q^{(1)}$ on the crack face are obtained.

(3) Next, the crack is again treated as a slit. Taking the extended stress fields $q^{(1)}$ on the crack faces obtained in Step (2) as the new boundary conditions and combining them with the boundary conditions in eqns (9) and (10), we obtain the extended displacement fields $\left(u_{i}^{(2)}, \theta^{(2)}\right)$ and a new crack cavity region $\Omega_{\mathrm{c}}^{(2)}$.

(4) Steps (2) and (3) are repeated until the preset precision for the thermal flux density on the crack face is satisfied at the $k$ th step, such that

$$
\left|\frac{q^{(k)}-q^{(k-1)}}{q^{(k-1)}}\right| \leq \delta,
$$

where $\delta$ is a preset error value. In the present paper, $\delta=0.01 \%$.

With this iterative method, we ultimately obtain the exact thermal boundary condition on the crack face and the size of the crack cavity. Simultaneously, the displacement field, temperature field, stress field and thermal flux density field of thermal-medium crack model under exact crack face boundary conditions are obtained.

\subsection{Sub-region BEM for a crack in a thermoelastic solid}

Employing the fundamental solutions and the Somigliana identity, the extended displacement boundary integral equations for the three sub-regions are established.

For thermoelastic solids, when the temperature in the solid is changed, an initial strain occurs owing to the thermal expansion. This initial strain is given by

$$
\varepsilon_{j k}^{0}=\alpha \Delta T \delta_{j k},
$$

where $\alpha$ denotes the thermal expansion coefficient, $\Delta T$ denotes the variations in temperature and $\delta$ denotes the Kronecker delta function. Eqn (14) indicates that the temperature induces a purely volume expansion. Further, the initial stress caused by the temperature field in the solids is given by

$$
\sigma_{j k}^{0}=2 G \frac{1+v}{1-2 v} \alpha \Delta T \delta_{j k} .
$$

For the sub-regions $\Omega_{1}$ and $\Omega_{2}$, the fundamental solutions are identical. Regarding the initial temperature stress as the body force, the extended displacement boundary integral equation 
for an arbitrary point $Y$ in a thermal solid with a body force $f_{j}$ within domain $\Omega_{1}$ or $\Omega_{2}$ can be written as

$$
u_{i}(Y)=\int_{\Gamma}\left[U_{i j}^{*}(x, Y) t_{j}(x)-T_{i j}^{*}(x, Y) u_{j}(x)\right] d \Gamma(x)+\int_{\Omega} U_{i j}^{*}(x, Y) f_{j} d \Omega,
$$

where $x$ is the field point; $j=1,2,3 ; u_{3}=\theta ; t_{3}=q ; f_{1}=f_{2}=0$ and $f_{3}=\sigma_{i j}{ }^{0} ; \Gamma=\Gamma_{1}$ or $\Gamma_{2}$; and $\Omega=\Omega_{1}$ or $\Omega_{2}$. Finally, $U_{i j}{ }^{*}$ and $T_{i j}{ }^{*}$ are the extended displacement and traction matrices of the fundamental solutions, which can be expressed as in [21]

$$
\begin{aligned}
& U_{i j}^{*}(x, Y)=-\frac{1}{8 \pi \mu(1-v)}\left[(3-4 v) \ln (r) \delta_{i j}-r_{, i} r_{, j}\right], \\
& T_{i j}^{*}(x, Y)=\frac{1}{4 \pi(1-v)} \frac{1}{r}\left\{(1-2 v)\left(r_{, i} n_{j}-r_{, j} n_{i}\right)-r_{, n}\left[(1-2 v) \delta_{i j}+2 r_{, i} r_{, j}\right]\right\},
\end{aligned}
$$

where xi and Yi denote the coordinates of the field point and source point, respectively; and the radius vector $\vec{r}$ between the source point and field point is defined as

$$
\begin{aligned}
& r_{, i}=\frac{\partial r}{\partial x_{i}}=\frac{r_{i}}{r}, \quad r_{, n}=\frac{\partial r}{\partial n}=r_{, i} n_{i}, \\
& r_{i}=x_{i}-Y_{i}, \quad r=\sqrt{r_{i} r_{i}} .
\end{aligned}
$$

When $Y$ approaches the boundary $\Gamma$, eqn (16) becomes

$$
C_{i j}(Y) u_{i}(Y)=\int_{\Gamma}\left[U_{i j}^{*}(x, Y) t_{j}(x)-T_{i j}^{*}(x, Y) u_{j}(x)\right] d \Gamma(x)+\int_{\Omega} U_{i j}^{*}(x, Y) f_{j} d \Omega,
$$

where $C_{i j}$ is the boundary coefficient matrix wherein $C_{i j}=\delta_{i j} / 2$ when the boundary is smooth. Employing the Gauss divergence theorem, the domain integral in eqn (19) can be transformed into the boundary integral

$$
\int_{\Omega} U_{i j}^{*}(x, Y) f_{j} d \Omega=\int_{\Gamma}\left[\left(R_{i}(x, Y) \theta(x)-Q_{i}(x, y) \frac{\partial \theta(x)}{\partial n}\right] d \Gamma,\right.
$$

where $\partial \theta(x) / \partial n$ denotes the normal temperature gradients of the temperature $\theta(x)$ on the boundary $\Gamma$. Further, $R_{i}(x, Y)$ and $Q_{i}(x, Y)$ are given by [21]

$$
\begin{aligned}
& R_{i}(x, Y)=\frac{\alpha(1+v)}{4 \pi(1-v)}\left[\left(\ln \frac{1}{r}-\frac{1}{2}\right) n_{i}-r_{, i} r_{, n}\right], \\
& Q_{i}(x, Y)=\frac{\alpha(1+v)}{4 \pi(1-v)}\left(\ln \frac{1}{r}-\frac{1}{2}\right) r_{i},
\end{aligned}
$$

whereupon the extended displacement boundary integral equation can be given by

$$
C_{i j}(Y) u_{i}(Y)=\int_{\Gamma}\left[\begin{array}{c}
U_{i j}^{*}(x, Y) t_{j}(x)-T_{i j}^{*}(x, Y) u_{j}(x) \\
+R_{i}(x, Y) \theta(x)-Q_{i}(x, Y) \frac{\partial \theta(x)}{\partial n}
\end{array}\right] d \Gamma(x) .
$$

For the sub-regions $\Omega_{\mathrm{c}}$, eqn (8) can be treated as a potential problem, whereby the integral equation for the temperature of any arbitrary point $Y$ within the crack cavity $\Omega_{c}$ is given by 


$$
\theta(Y)=\int_{\Gamma}\left[\Phi^{*}(x, Y) \frac{\partial \theta(x)}{\partial n}-\frac{\partial \Phi^{*}(x, Y)}{\partial n} \theta(x)\right] d \Gamma(x)
$$

where

$$
\Phi^{*}(x, Y)=\frac{1}{2 \pi} \ln \frac{1}{r}, \quad \frac{\partial \Phi^{*}(x, Y)}{\partial n}=-\frac{1}{2 \pi r} r_{, i} n_{i} .
$$

When $Y$ approaches the boundary $\Gamma$, eqn (23) becomes

$$
C(Y) \theta(Y)=\int_{\Gamma}\left[\Phi^{*}(x, Y) \frac{\partial \theta(x)}{\partial n}-\frac{\partial \Phi^{*}(x, Y)}{\partial n} \theta(x)\right] d \Gamma(x) .
$$

When the crack is treated as a slit, only two sub-regions exist; i.e., $\Omega_{1}$ and $\Omega_{2}$. Using linear elements to discretize the boundaries of $\Omega_{1}$ and $\Omega_{2}$, the total linear system of algebraic equations for all nodes on the boundaries $\Gamma_{1}$ and $\Gamma_{2}$ can be written in the matrix form

$$
\left[\begin{array}{cc}
\mathbf{A}^{(1)} & 0 \\
0 & \mathbf{A}^{(2)}
\end{array}\right]\left[\begin{array}{l}
\mathbf{u}^{(1)} \\
\mathbf{u}^{(2)}
\end{array}\right]=\left[\begin{array}{cc}
\mathbf{B}^{(1)} & 0 \\
0 & \mathbf{B}^{(2)}
\end{array}\right]\left[\begin{array}{l}
\mathbf{t}^{(1)} \\
\mathbf{t}^{(2)}
\end{array}\right],
$$

where $\mathbf{u}^{(1)}$ and $\mathbf{u}^{(2)}$ denote the extended displacement fields $\left(u_{i}, \theta\right)$ of $\Omega_{1}$ and $\Omega_{2}$, respectively; and $\mathbf{t}^{(1)}$ and $\mathbf{t}^{(2)}$ denote the extended stress fields $\left(\sigma_{i j}, h_{i}\right)$ of $\Omega_{1}$ and $\Omega_{2}$, respectively. The compatibility conditions are given as

$$
\mathbf{u}_{c b}^{(1)}=\mathbf{u}_{c b}^{(2)}, \quad \mathbf{t}_{c b}^{(1)}=-\mathbf{t}_{c b}^{(2)},
$$

where $\mathbf{u}_{\mathrm{cb}}{ }^{(1)}$ and $\mathbf{u}_{\mathrm{cb}}{ }^{(2)}$ denote the displacement fields $\left(u_{i}, \theta\right)$ on the common boundaries of $\Omega_{1}$ and $\Omega_{2}$, respectively; and $\mathbf{t}_{\mathrm{cb}}{ }^{(1)}$ and $\mathbf{t}_{\mathrm{cb}}{ }^{(2)}$ denote the extended stress fields $\left(\sigma_{i j}, h_{i}\right)$ of the common boundaries of $\Omega_{1}$ and $\Omega_{2}$, respectively. Substituting the boundary conditions given in eqns (9)-(11) and the compatibility conditions given in eqn (27) into eqn (26), the unknown extended displacements $\left(u_{i}, \theta\right)$ and the extended stress fields $\left(\sigma_{i j}, h_{i}\right)$ on the boundaries $\Gamma_{1}, \Gamma_{2}$ and common boundaries $\Gamma_{12}\left(\Gamma_{21}\right)$ can be obtained.

Similarly, in the crack cavity $\Omega_{\mathrm{c}}$, the total linear system of algebraic equations for all nodes on the crack face can be written in the matrix form

$$
\mathbf{H} \boldsymbol{\theta}^{c}=\mathbf{G h}^{c},
$$

where $\theta^{c}$ and $\mathbf{h}^{c}$ respectively denote the temperature fields and the thermal flux density fields in the crack cavity $\Omega_{\mathrm{c}}$. Substituting the boundary conditions given in eqn (11) into eqn (28), the unknown temperature field $\theta$ and the thermal flux density $h$ in the crack cavity $\Omega_{\mathrm{c}}$ are obtained.

\section{NUMERICAL ANALYSIS: A CENTRAL CRACK IN A RECTANGULAR THERMOELASTIC PLATE}

The material of the plate was $\mathrm{ZrO}_{2}$ ceramic, with the material constants

$$
\begin{aligned}
& E=200 \times 10^{9} \mathrm{~N} / \mathrm{m}^{2}, \quad v=0.29, \\
& \alpha=10.6 \times 10^{-6} \mathrm{~m} / \mathrm{K}, \quad \beta=4.2 \mathrm{~W} /(\mathrm{m} . \mathrm{K}) .
\end{aligned}
$$

The iterative sub-region BEM proposed in Section 3 was used to analyze the central crack model. 


\subsection{Validation of the iterative BEM}

The plate dimensions used were a height $2 h=100 \mathrm{~mm}$ and width $2 l=100 \mathrm{~mm}$, and a central crack length $2 c=10 \mathrm{~mm}$. Further, the extended external loadings were

$$
p_{0}=100 \mathrm{MPa}, \quad q_{0}=0.1 \mathrm{~W} / \mathrm{m}^{2} .
$$

In this case, we assumed that the central crack in the thermal plate was thermally impermeable, the iteration times satisfying the accuracy requirement in eqn (13) are ten. The calculated crack opening displacement (COD) and the temperature across the crack face were in good agreement with those obtained by the finite element method (FEM), as shown in Figs 3 and 4. This comparison of the results demonstrates that the proposed method is effective for fracture analysis of thermoelastic solids with a central crack.

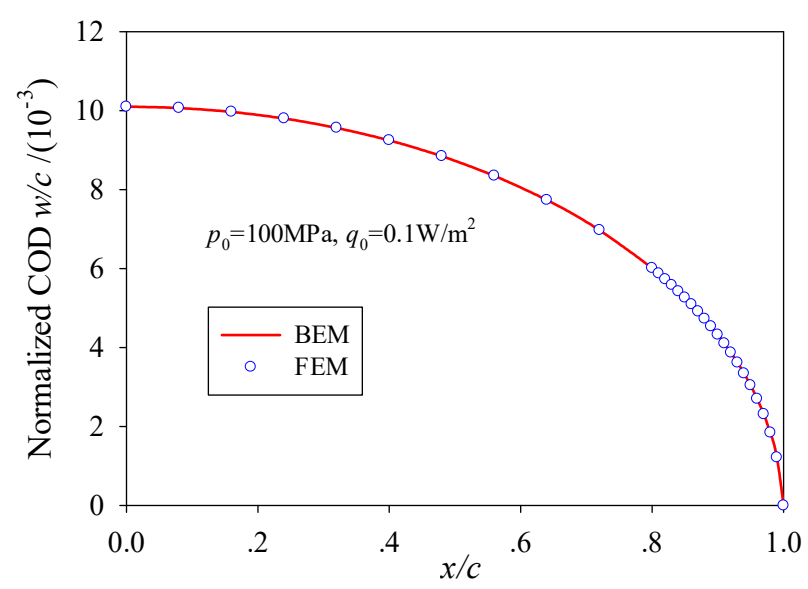

Figure 3: Normalized crack opening displacement (COD) $w / c$ across the crack face under a mechanical loading of $p_{0}=100 \mathrm{MPa}$ and thermal loading of $q_{0}=0.1 \mathrm{~W} / \mathrm{m}^{2}$.

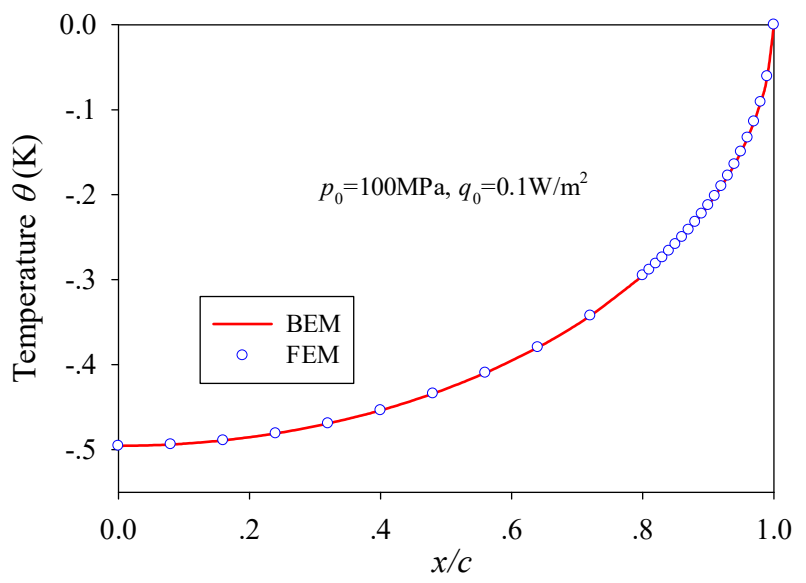

Figure 4: Temperature $\theta$ across the crack face under a mechanical loading of $p_{0}=100 \mathrm{MPa}$ and thermal loading of $q_{0}=0.1 \mathrm{~W} / \mathrm{m}^{2}$. 
4.2 Effect of crack length on the extended displacements of a central crack in a thermoelastic solid

As an example, we studied the effect of the crack size on the COD and the temperature across the crack face. In this case, the ratio of the plate height to crack length $h / c$ was fixed as 10 , the crack face possessed impermeable boundary conditions, and the external mechanical loading $p_{0}$ and thermal loading $q_{0}$ were the same as given in eqn (30).

The COD and temperature across the crack face are plotted in Figs 5 and 6, respectively. It can be seen that, with increasing crack length, the COD increased while the temperature across the crack face decreased.

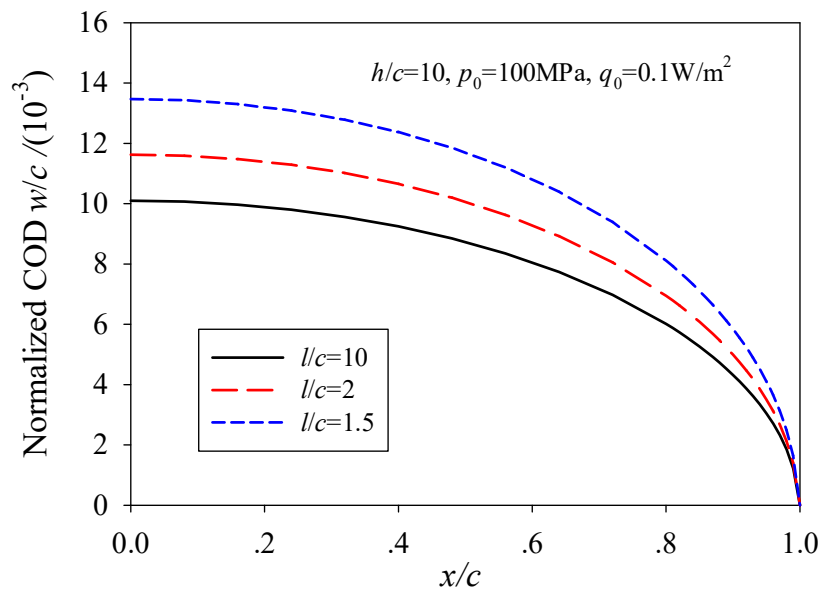

Figure 5: Normalized crack opening displacement (COD) $w / c$ across the crack face with various $l / c$ and fixed $h / c=10$ under a mechanical loading of $p_{0}=100 \mathrm{MPa}$ and thermal loading of $q_{0}=0.1 \mathrm{~W} / \mathrm{m}^{2}$.

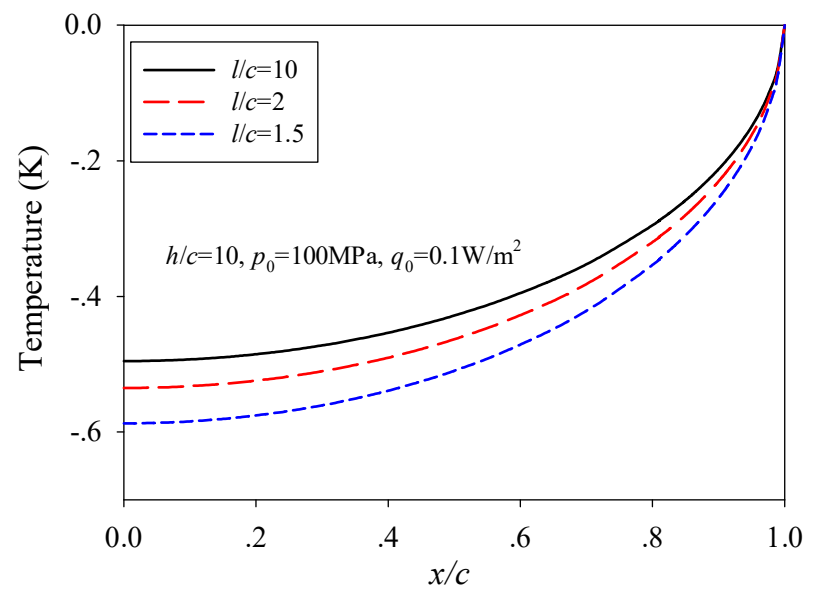

Figure 6: Temperature $\theta$ across the crack face with various $l / c$ and fixed $h / c=10$ under a mechanical loading of $p_{0}=100 \mathrm{MPa}$ and thermal loading of $q_{0}=0.1 \mathrm{~W} / \mathrm{m}^{2}$. 
4.3 Effects of extended loads on the extended stress intensity factors of a central crack in a thermoelastic solid

Using the proposed iterative BEM, the crack opening displacements and temperatures near the crack tip were obtained. Then, the stress intensity factor $K_{\mathrm{I}}$ and thermal flux density intensity factor $K_{\mathrm{T}}$ near the crack tip were calculated using [19]

$$
K_{\mathrm{I}}=\frac{E}{8\left(1-v^{2}\right)} \lim _{\rho \rightarrow 0} \sqrt{\frac{2 \pi}{\rho}}\|w\|, \quad K_{\mathrm{T}}=-\frac{\beta}{4} \lim _{\rho \rightarrow 0} \sqrt{\frac{2 \pi}{\rho}}\|\theta\|, \quad\left(\|w\|=w^{+}-w^{-},\|\theta\|=\theta^{+}-\theta^{-}\right),
$$

where $\rho$ is the distance between a point on the crack face and the crack tip; $\|w\|$ is the displacement discontinuity across the crack face; and $\|\theta\|$ is the temperature discontinuity across the crack face; whose subscripts "+" and "-" denote the upper and lower crack faces, respectively. In our numerical calculation, $\|w\|$ and $\|\theta\|$ were obtained by fitting the corresponding values for two elements close to the crack tip, and the normalized stress intensity factor and heat flux density intensity factor are given by

$$
F_{\mathrm{I}}=K_{\mathrm{I}} /\left(p_{0} \sqrt{\pi c}\right), \quad F_{\mathrm{T}}=K_{\mathrm{T}} /\left(q_{0} \sqrt{\pi c}\right) .
$$

In the following calculation, the geometric parameters $h / c$ and $l / c$ were fixed as 10 . The crack face boundary conditions are the exact boundary condition as in eqn (11) and the external mechanical loading $p_{0}$ and thermal loading $q_{0}$ are the same as that in eqn. (30).

It can be observed that the normalized stress intensity factors $F_{\text {I }}$ and thermal flux intensity factor $F_{\mathrm{T}}$ remain the same with the increase of the normalized mechanical loading $p / p_{0}$, as shown in Fig. 7.

It is found that the normalized thermal flux intensity factor $F_{\mathrm{T}}$ remains constant with increasing normalized thermal loading $q / q_{0}$. The normalized stress intensity factor $F_{\mathrm{I}}$, however, increases with increasing normalized thermal loading $q / q_{0}$, as shown in Fig. 8.

We can see in Figs 7 and 8 that a one-way coupling property exists in the thermoelastic solids. Specifically, for the thermoelastic solids, the mechanical characteristic does not affect the thermal characteristic, while the thermal characteristic does affect the mechanical characteristic.

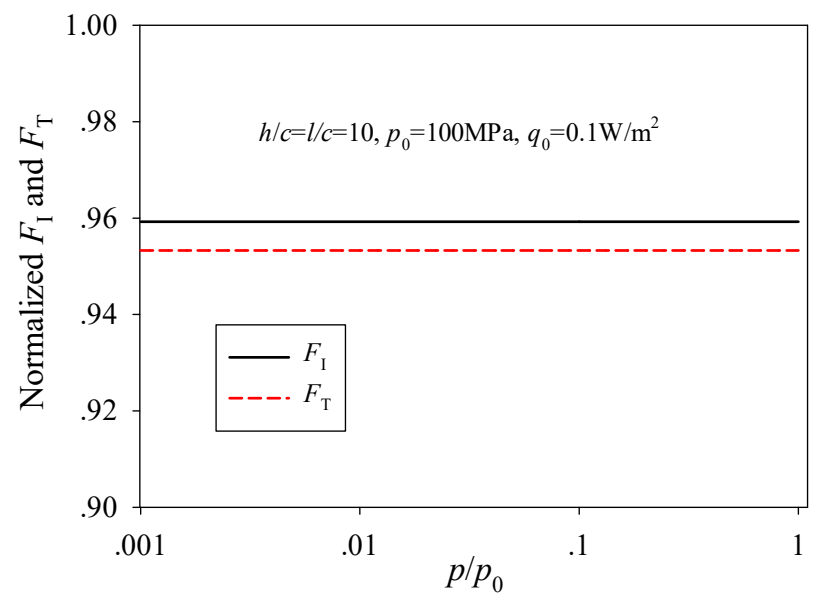

Figure 7: Normalized intensity factors $F_{\mathrm{I}}$ and $F_{\mathrm{T}}$ versus normalized $p / p_{0}$ with $h=l=10 c$, under a mechanical loading of $p_{0}=100 \mathrm{MPa}$ and thermal loading of $q_{0}=0.1 \mathrm{~W} / \mathrm{m}^{2}$. 


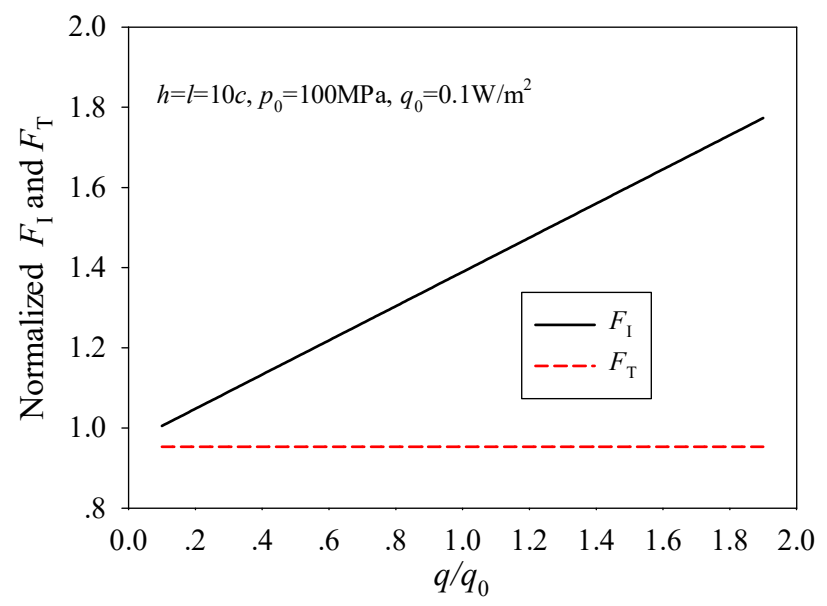

Figure 8: Normalized intensity factors $F_{\mathrm{I}}$ and $F_{\mathrm{T}}$ versus normalized $q / q_{0}$ with $h=l=10 c$, under a mechanical loading of $p_{0}=100 \mathrm{MPa}$ and thermal loading of $q_{0}=0.1 \mathrm{~W} / \mathrm{m}^{2}$.

\section{CONCLUSIONS}

An iterative BEM was proposed to analyze the cracking in thermoelastic solids using exact crack boundary conditions. The proposed method was validated by comparing the COD and temperature across the crack face with the results obtained by FEM. Numerical results demonstrated that the crack size greatly influences the COD and temperature across the crack face and that a one-way mechanical-thermal coupling property exists in thermoelastic solids. The proposed iterative BEM is an efficient approach for solving the fracture problem of thermoelastic solids.

\section{ACKNOWLEDGEMENT}

The work was supported by the National Natural Science Foundation of China (Nos. $11572289,11702251)$.

\section{REFERENCES}

[1] Nowacki, W., Thermoelasticity, Pergamon Press: New York, 1962.

[2] Khaund, A.K., Krstic, V.D. \& Nicholson, P.S., Influence of elastic and thermal mismatch on the local crack-driving force in brittle composites. Journal of Materials Science, 12(11), pp. 2269-2273, 1977. DOI: 10.1007/bf00552248.

[3] Noda, N. \& Jin, Z.H., Thermal stress intensity factors for a crack in a strip of a functionally gradient material. International Journal of Solids and Structures, 30(8), pp. 1039-1056, 1993. DOI: 10.1016/0020-7683(93)90002-o.

[4] Erdogan, F. \& Wu, B.H., Crack problems in FGM layers under thermal stresses. Journal of Thermal Stresses, 19(3), pp. 237-265, 1996.

DOI: $10.1080 / 01495739608946172$.

[5] Chen, J., Soh, A.K., Liu, J. \& Liu, Z., Thermal fracture analysis of a functionally graded orthotropic strip with a crack. International Journal of Mechanics and Materials in Design, 1(2), pp. 131-141, 2004.

[6] Chen, Z.T. \& Hu, K.Q., Thermo-elastic analysis of a cracked half-plane under a thermal shock impact using the hyperbolic heat conduction theory. Journal of Thermal Stresses, 35(4), pp. 342-362, 2012. DOI: 10.1080/01495739.2012.663685. 
[7] Dang, H.Y., Zhao, M.H., Fan, C.Y. \& Chen, Z.T., Analysis of an arbitrarily shaped interface crack in a three-dimensional isotropic thermal elastic bi-material. Part 2: Numerical method. International Journal of Solids and Structures, 99, pp. 48-56, 2016. DOI: 10.1016/j.ijsolstr.2016.08.007.

[8] Wang, P., Wang, K.F., Wang, B.L. \& Cui, Y.J., Effective thermoelectric conversion properties of thermoelectric composites containing a crack/hole. Composite Structures, 191, pp. 180-189, 2018. DOI: 10.1016/j.compstruct.2018.02.049.

[9] Yu, X.M., Shah, K. \& Mukherjee, S., Thermoelastic fracture mechanics with regularized hypersingular boundary integral equations. Engineering Analysis with Boundary Elements, 23(1), pp. 89-96, 1999. DOI: 10.1016/s0955-7997(98)00064-2.

[10] Shiah, Y.C. \& Tan, C.L., Exact boundary integral transformation of the thermoelastic domain integral in BEM for general 2D anisotropic elasticity. Computational Mechanics, 23(1), pp. 87-96, 1999. DOI: 10.1007/s004660050387.

[11] Duflot, M., The extended finite element method in thermoelastic fracture mechanics. International Journal for Numerical Methods in Engineering, 74(5), pp. 827-847, 2008. DOI: 10.1002/nme.2197.

[12] Sladek, J., Sladek, V., Solek, P. \& Zhang, C.Z., Two- and three-dimensional transient thermoelastic analysis by the MLPG method. Computer Modeling in Engineering and Sciences, 47(1), pp. 61-95, 2009.

[13] Brebbia, C.A., The Boundary Element Method for Engineers, Halsted Press: New York, 1978.

[14] Portela, A., Aliabadi, M.H. \& Rooke, D.P., The dual boundary element method: Effective implementation for crack problems. International Journal for Numerical Methods in Engineering, 33(6), pp. 1269-1287, 2010.

DOI: $10.1002 /$ nme.1620330611.

[15] Prasad, N.N.V., Aliabadi, M.H. \& Rooke, D.P., The dual boundary element method for thermoelastic crack problems. International Journal of Fracture, 33(3), pp. 255272, 1994. DOI: $10.1007 / \mathrm{bf00042588.}$

[16] Ekhlakov, A., Khay, O., Zhang, C., Sladek, J., Sladek, V. \& Gao, X.W., Thermoelastic crack analysis in functionally graded materials and structures by a BEM. Fatigue and Fracture of Engineering Materials and Structures, 35(8), pp. 742-766, 2012. DOI: 10.1111/j.1460-2695.2011.01657.x.

[17] Pasternak, I., Boundary integral equations and the boundary element method for fracture mechanics analysis in 2D anisotropic thermoelasticity. Engineering Analysis with Boundary Elements, 36(12), pp. 1931-1941, 2012.

DOI: 10.1016/j.enganabound.2012.07.007.

[18] Zhao, M.H., Dang, H.Y., Li, Y., Fan, C.Y. \& Xu, G.T., Displacement and temperature discontinuity boundary integral equation and boundary element method for analysis of cracks in three-dimensional isotropic thermoelastic media. International Journal of Solids and Structures, 81, pp. 179-187, 2016. DOI: 10.1016/j.ijsolstr.2015.11.024.

[19] Zhong, X.C. \& Kang, Y.L., A thermal-medium crack model. Mechanics of Materials, 51, pp. 110-117, 2012. DOI: 10.1016/j.mechmat.2012.04.013.

[20] Zhong, X.C., Long, X.Y. \& Zhang, L.H., An extended thermal-medium crack model. Applied Mathematical Modelling, 56, pp. 202-216, 2018.

DOI: 10.1016/j.apm.2017.11.016.

[21] Gao, X.W., Boundary element analysis in thermoelasticity with and without internal cells. International Journal for Numerical Methods in Engineering, 57(7), pp. 975990, 2003. DOI: 10.1002/nme.715. 